\title{
PERFIL SOCIODEMOGRÁFICO E PERCEPÇÃO DOS CONSUMIDORES SOBRE A SEGURANÇA ALIMENTAR DE PRODUTOS LÁCTEOS DE ORIGENS BUBALINA E BOVINA EM MUNICÍPIOS DO ARQUIPÉLAGO DO MARAJÓ, ESTADO DO PARÁ
}

\author{
Andrea Viana da $\mathrm{Cruz}^{1}$ \\ Elton Alex Correa da Silva ${ }^{2}$ \\ Allana Lais Alves Lima ${ }^{3}$ \\ Iroleide Santana de Jesus ${ }^{1}$ \\ Regiane Ferreira Feitosa ${ }^{1}$ \\ Mylla Christy da Silva Dufossé ${ }^{4}$ \\ Ana Lídia de Brito Oliveira ${ }^{5}$ \\ Rafael Monteiro de Melo ${ }^{5}$ \\ Adrianne Maria Brito Pinheiro da Rosa ${ }^{4}$ \\ Gabriela Sampaio ${ }^{6}$ \\ Gabriela Sousa Silva ${ }^{6}$ \\ Ana Paula Presley Oliveira Sampaio ${ }^{4}$ \\ Francisco Rômulo Oliveira Magalhães ${ }^{1}$ \\ Joelson Sousa Lima ${ }^{4}$ \\ Talita Bandeira Roos ${ }^{7}$ \\ Carina Martins de Moraes ${ }^{8}$
}

\begin{abstract}
RESUMO
O objetivo deste estudo foi caracterizar o perfil do consumidor de produtos lácteos de origens bubalina e bovina nos municípios de Soure e Salvaterra (Ilha do Marajó-PA) quanto ao perfil sociodemográfico, ao nível de conhecimento sobre qualidade higiênico-sanitária e sobre fraudes nesses produtos. Para coleta dos dados, foram aplicados questionários a voluntários escolhidos ao acaso. Os dados foram analisados de forma descritiva e, posteriormente, submetidos à análise de correspondência múltipla (ACM). A coleta de dados resultou em 403 entrevistas. Os resultados demonstraram que os consumidores de produtos de origem bubalina eram, em sua maioria, do sexo masculino, com idade acima de 40 anos, que consomem principalmente de produtores rurais, enquanto que os consumidores de produtos bovinos eram mulheres com idades entre 21 e 30 anos, sendo os alimentos procedentes de estabelecimentos comerciais como supermercados e padarias.
\end{abstract}

Palavras-chave: bubalino, consumo, bovino, leite, produto rural.

\footnotetext{
${ }^{1}$ Mestre em Saúde Animal, Universidade Federal do Pará. Correspondência andrea.vianacruz@ gmail.com

${ }^{2}$ Mestrando no Programa de Pós-Graduação em Aquicultura e Recursos Aquáticos Tropicais (PPGAqRAT), Universidade Federal Rural da Amazônia - UFRA. eltonpesc@gmail.com.

3 Doutoranda em Reprodução Animal na Amazônia, Universidade Federal Rural da Amazônia. allana.lima1 @ hotmail.com.

${ }_{5}^{4}$ Doutoranda em Saúde Animal na Amazônia, Universidade Federal do Pará.

${ }^{5}$ Mestrando(a) em Saúde Animal na Amazônia, Universidade Federal do Pará. ana_oliveira@ hotmail.com.

${ }^{6}$ Graduando em Medicina Veterinária, Universidade Federal do Pará.

${ }^{7}$ Professor Adjunto da Universidade Federal do Pará. talitaroos@ gmail.com.

${ }^{8}$ Professora Associado II. Universidade Federal do Pará. carina_moraes@ terra.com.br.
}

Cruz AV, Silva EAC, Lima ALA, Jesus IS, Feitosa RF, Dufossé MCS, et al. Perfil sociodemográfico e percepção dos consumidores sobre a segurança alimentar de produtos lácteos de origens bubalina e bovina em municípios do Arquipélago do Marajó, estado do Pará. Vet. e Zootec. 2020.; 27: 001-014. 


\title{
SOCIODEMOGRAPHIC PROFILE AND CONSUMERS' PERCEPTION OF FOOD SAFETY FOR DAIRY PRODUCTS OF BUFFALO AND COW ORIGINS IN MUNICIPALITIES IN THE MARAJÓ ARCHIPELAGO, STATE OF PARÁ
}

\begin{abstract}
The aim of this study was to characterize the profile of consumers of dairy products of buffalo and cow origins in the municipalities of Soure and Salvaterra (Ilha do Marajó-PA), regarding the sociodemographic profile, the level of knowledge about hygiene and fraud in these products. For data collection, questionnaires were applied to volunteers chosen at random. The data were analyzed descriptively and subsequently submitted to multiple correspondence analysis (MCA). The data collection resulted in 403 interviews. The results showed that the consumers of products of buffalo origin were mostly male, aged over 40 years, who consume mainly from rural producers, while the consumers of bovine products were women aged between 21 and 30 years, with food coming from commercial establishments such as supermarkets and bakeries.
\end{abstract}

Key-words: buffalo, consumption, bovine, milk, rural product.

\section{PERFIL SOCIODEMOGRÁFICO Y PERCEPCIÓN DEL CONSUMIDOR SOBRE LA SEGURIDAD ALIMENTARIA DE PRODUCTOS LÁCTEOS DE ORIGENS BUFALINO Y BOVINO EN MUNICIPIOS DEL ARCHIPIÉLAGO DE MARAJÓ, ESTADO DE PARÁ}

\section{RESUMEN}

El objetivo de este estudio fue caracterizar el perfil de los consumidores de productos lácteos de origen bufalino y bovino en los municipios de Soure y Salvaterra (Ilha do Marajó-PA), con respecto al perfil sociodemográfico, el nivel de conocimiento sobre la calidad higiénicosanitaria y la existência de actos fraudulentos en estos productos. Para la recopilación de datos, se aplicaron cuestionarios a los voluntarios, elegidos al azar. Los datos fueron analizados descriptivamente y posteriormente sometidos a análisis de correspondencia múltiples (ACM). La recopilación de datos resultó en un total de 403 entrevistas. Los resultados mostraron que el consumidores de productos de origen búfalo eran en su mayoría hombres, mayores de 40 años, que consumían principalmente de productores rurales, mientras que los consumidores de productos bovinos estaban representados mayormente mujeres de 21 a 30 años, que consumían en establecimientos comerciales como supermercados y panaderías.

Palabras clave: bufalino, consumo, bovinos, leche, producto rural.

\section{INTRODUÇÃO}

O comportamento do consumidor consiste na procura por bens e serviços com os quais mantém uma relação e seus costumes estão relacionados com fatores como escolaridade, atividade profissional, cultura e lazer. Essas condições são essenciais para analisar o comportamento do consumidor, pois traduzem sua aceitação, resistência e hábitos de consumo (1,2). Nesse contexto, surge a importância do estudo do perfil do consumidor. Em geral, a determinação desse perfil exige o uso de análises estatísticas detalhadas, uma vez que, segundo Olsen (3), as atitudes ou motivações dos indivíduos são geralmente afetadas por um grupo complexo de fatores, onde a qualidade ou o valor não são as únicas razões para a 
compra ou consumo de certos produtos. O estabelecimento desse perfil em uma determinada região também é relevante quanto à percepção do consumidor sobre as condições higiênicosanitárias e acerca de fraudes em alimentos. Tendo em vista que essas questões permeiam o sistema de produção e afetam diretamente a população e, segundo Kendall et al. (4), geram riscos potenciais à saúde coletiva e dos consumidores vítimas de fraude alimentar, que podem vir a sofrer perdas financeiras e experimentar emoções negativas.

Entre os alimentos consumidos com frequência pelas mais diferentes populações, o leite e seus derivados merecem destaque. Esses Produtos de Origem Animal (POA) correspondem a um grupo alimentício de grande expressão para a nutrição humana, por conta de sua composição e nutrientes (5). Por esta razão, o conhecimento dos hábitos de consumo desses alimentos é relevante, embora o consumo de produtos lácteos de origem bovina seja amplamente estudado (6). No que se refere ao consumo de produtos bubalinos, poucas informações estão disponíveis no cenário nacional, embora existam trabalhos científicos relatando características dos consumidores de outros produtos como a carne (7). Estudos que busquem contribuir para a redução da escassez dessas informações são necessários.

O Arquipélago do Marajó possui aproximadamente 360.871 cabeças de búfalos, o que representa a maior concentração da espécie no Brasil segundo dados do Instituto Brasileiro de Geografia e Estatística-IBGE (8), fato que pode influenciar diretamente os hábitos alimentares dos habitantes do local. Além disso, o referido Arquipélago é um polo turístico, recebendo pessoas de diversos países, devendo-se ressaltar que a gastronomia apresenta papel de destaque em toda a ilha, devido à venda de produtos de origem bubalina. Um dos produtos mais reconhecidos é o queijo do Marajó, produzido do leite de búfala (9), que merece destaque e corresponde a uma indicação geográfica com valor histórico, cultural e econômico (10). Visto isto, traçar o perfil de uma parcela dessa comunidade com hábitos alimentares e cultural, voltadas para a produção bubalina é essencial, tanto para a comunidade acadêmica ter base para desenvolver novas pesquisas quanto para a cadeia produtiva do búfalo. Diante disso, o presente estudo visa caracterizar o perfil do consumidor de produtos lácteos de origens bubalina e bovina nos municípios de Soure e Salvaterra (Ilha do Marajó-PA), quanto ao perfil sociodemográfico, ao nível de conhecimento sobre qualidade higiênico-sanitária e fraudes nesses alimentos.

\section{MATERIAL E MÉTODOS}

Este estudo foi submetido à plataforma Brasil e aprovado pelo Comitê de Ética em Pesquisa - CEP do Instituto de Ciências da Saúde da Universidade Federal do Pará (parecer $\mathrm{n}^{\circ}$ 3.532.655). Os pesquisadores responsabilizaram-se em proceder conforme as normas recomendadas pela Resolução do Conselho Nacional de Saúde-CNS 466/12 (11), enquanto que os respondentes, ao aceitarem participar da pesquisa, foram elucidados quanto ao objetivo, modo de aplicação e destino dos dados, tendo sido convidados, a seguir, a assinar o Termo de Consentimento Livre e Esclarecido (TCLE), no intuito de garantir que nenhum participante fosse submetido aos mecanismos de coleta dos dados sem receber as devidas orientações e sem assinar o TCLE.

Para o cálculo do tamanho amostral foi utilizado o software openepi, disponível no site de domínio público www.openepi.com (12). Para as bases do cálculo foi levado em consideração a população dos dois municípios e as medidas de precisão, sendo o intervalo de confiança de $95 \%$, erro padrão de $\pm 5 \%$ e frequência antecipada de $50 \%$, a partir da aplicação da seguinte equação:

$$
\begin{aligned}
& n=\left[\mathrm{EDFF}^{*} \mathrm{~Np}(1-\mathrm{p})\right] /\left[\left(\mathrm{d}^{2} / \mathrm{Z}_{1-\alpha / 2}^{2} *(\mathrm{~N}-1)+\mathrm{p}^{*}(1-\mathrm{p})\right]\right. \\
& \text { Onde: } \\
& \mathrm{N} \text { : Tamanho da população }
\end{aligned}
$$

Cruz AV, Silva EAC, Lima ALA, Jesus IS, Feitosa RF, Dufossé MCS, et al. Perfil sociodemográfico e percepção dos consumidores sobre a segurança alimentar de produtos lácteos de origens bubalina e bovina em municípios do Arquipélago do Marajó, estado do Pará. Vet. e Zootec. 2020.; 27: 001-014. 
( $p)$ : frequência \% hipotética do fator do resultado na população

$(d)$ : Limites de confiança

EDFF: Efeito de desenho

O presente estudo qualitativo possui caráter descritivo e foi realizado por meio de aplicação de questionário com perguntas fechadas, elaborado com adaptações das questões descritas por Vidal-Martins et al. (13), sendo abordados aspectos relacionados ao perfil e hábitos de consumo de produtos lácteos bubalinos e bovinos. A caracterização do perfil demográfico dos entrevistados foi realizada por perguntas como: localidade onde reside, escolaridade, sexo e faixa etária.

As entrevistas ocorreram nos municípios de Salvaterra e Soure, na ilha de Marajó-PA, nas ruas que compõem o centro comercial da cidade, onde há o maior fluxo de pessoas. Todos que participaram da pesquisa foram abordados ao acaso, sem distinção de sexo ou raça, apresentando como caráter excludente apenas a faixa etária, pois somente pessoas maiores de 18 anos foram abordadas pela equipe. Durante o período de realização da pesquisa foram entrevistados 403 indivíduos, sendo 170 em Salvaterra e 233 em Soure.

Após a coleta das informações, os dados apurados foram tabulados em forma de matriz qualitativa em planilha eletrônica no software Microsoft Office Excel 2016® e, em seguida, transformados em frequência relativa. Para as inferenciais utilizou-se o teste do Qui-quadrado de associação, com nível de significância $\mathrm{p} \leq 0,05$, gerados no pacote estatístico PAST for Windows versão 3.26 de 2019. Para as análises de correspondência múltipla (ACM), análises que permitem identificar as associações entre as características dos consumidores dos municípios de Salvaterra e Soure, foi utilizado o software R Studio $\odot$ (FactoextraR, FactoMineR).

\section{RESULTADOS E DISCUSSÃO}

A Tabela 1 apresenta o perfil demográfico dos entrevistados nos municípios de Salvaterra e Soure.

Quando questionados quanto ao consumo de leite e produtos lácteos, observou-se que 98,2\% (167/170) dos entrevistados em Salvaterra e 98,3\% (229/233) em Soure afirmaram consumir, enquanto 1,25\% (3/170) em Salvaterra e 1,7\% (4/233) em Soure declararam não consumir tais produtos. Este fato também foi relatado por Matiuzzo \& Silva (5) que, ao caracterizarem o consumo de leite em Campos de Júlio-MT, observaram que $86,7 \%$ dos entrevistados consumiam leite, e por Pereira et al. (14) que, ao investigarem o perfil dos consumidores de leite do Município de Maringá-PR, constataram que 46,5\% consumiam o produto. Esses resultados demonstram a importância do comércio de leite no Brasil, mesmo em regiões sem tradição na produção leiteira, como o Arquipélago do Marajó.

Observou-se participação semelhante de entrevistados pertencentes ao sexo masculino e feminino, uma vez que 53\% (90/170) dos indivíduos analisados em Salvaterra e 54\% (126/ 233) em Soure declararam ser do sexo masculino, enquanto que 47\% (80/170) e 46\% (107/ 233) em Salvaterra e Soure respectivamente são do sexo feminino (Tabela 1). Os dados obtidos em relação ao sexo dos entrevistados contrastam com o observado por Matiuzzo e Silva (5) onde $85,8 \%$ dos entrevistados eram do sexo feminino.

Quanto à faixa etária, em Salvaterra predominaram idades acima de 40 anos, representando $33 \%(56 / 170)$, os demais declararam ser menores de 20 , representando $10 \%$ (18/170), verificou-se também que $31 \%(52 / 170)$ tinham entre 21 a 30 anos e $26 \%(44 / 170)$ entre 31 a 40 anos (Tabela 1). Resultados similares foram observados em Soure, onde 43\% (99/233) afirmaram ter acima de 40 anos, 8\% (19/233) declararam ser menores de 20 anos, $27 \%(64 / 233) 21$ a 30 anos e 22\% (51/233) entre 31 a 40 anos. Esses resultados diferem dos encontrados por Marciana et al. (15) que em um estudo, avaliaram o conhecimento da 
comunidade de São Luís de Montes Belos-Goiás sobre o consumo de produtos sem lactose em derivados lácteos, demostraram que a faixa etária de maior expressão foi a de 16 a 25 anos, representando $69 \%$ dos entrevistados. Esses resultados podem estar relacionados ao fato dos grupos amostrais não serem homogêneos.

Tabela 1. Frequência de ocorrência e relativa do perfil sociodemográfico dos entrevistados em Soure e Salvaterra.

\begin{tabular}{cccccc}
\hline \multirow{2}{*}{ Pergunta } & Categoria & F.O & F.R $(\boldsymbol{\%})$ & & \\
\cline { 2 - 6 } & & Soure & Salvaterra & Soure & Salvaterra \\
\hline \multirow{2}{*}{ Sexo } & Masculino & 126 & 90 & 54.08 & 52.94 \\
& Feminino & 107 & 80 & 45.92 & 47.06 \\
Onde Mora & Urbana & 199 & 150 & 85.41 & 88.24 \\
& Rural & 34 & 20 & 14.59 & 11.76 \\
& $20<$ & 19 & 18 & 8.15 & 10.59 \\
Faixa etária & 20 a 30 & 64 & 52 & 27.47 & 30.59 \\
& 31 a 40 & 51 & 44 & 21.89 & 25.88 \\
& $>40$ & 99 & 56 & 42.49 & 32.94 \\
& Analfabeto & 0 & 1 & 0.00 & 0.59 \\
& Fun incompleto & 51 & 24 & 21.89 & 14.12 \\
& Fun completo & 31 & 35 & 13.30 & 20.59 \\
& Med incompleto & 29 & 26 & 12.45 & 15.29 \\
& Med completo & 86 & 43 & 36.91 & 25.29 \\
& Sup incompleto & 11 & 27 & 4.72 & 15.88 \\
& Sup completo & 25 & 14 & 10.73 & 8.24 \\
\hline
\end{tabular}

Em relação ao nível de escolaridade dos entrevistados, observou-se que em Salvaterra, $25 \%$ (43/ 170) declararam possuir ensino médio completo, $21 \%$ (31/ 170) fundamental completo, $16 \%$ (27/170) superior incompleto, 15\% (26/170) ensino médio incompleto, $14 \%$ (24/170) fundamental incompleto, 8\% (14/170) superior completo, e 1\% (1/170) era analfabeto. Em Soure, observou-se uma similaridade ao encontrado em Salvaterra, onde o ensino médio completo foi o nível escolar mais expressivo, sendo 37\% (86/233) entre os entrevistados, seguido por $22 \%$ (51/233) com fundamental incompleto, 14\% (33/ 233) fundamental completo, $12 \%(29 / 233)$ ensino médio incompleto, $11 \%$ (25/233) superior completo, e 5\% (11/233), superior incompleto (Tabela 1).

Estes resultados são similares aos descritos por Matiuzzo e Silva (5), que verificaram que $35,8 \%$ dos indivíduos possuíam ensino médio completo, no entanto são distintos dos apresentados por Marciana et al. (15), pois dentre os entrevistados o nível escolar mais expressivo foi ensino superior incompleto, com 61,6\%. Ainda, em um estudo realizado por Ferreira et al. (6), que realizaram um levantamento do perfil e hábitos dos consumidores do leite visando identificar o consumidor de leite in natura (cru), observaram que 40,3\% dos entrevistados possuíam o primeiro grau incompleto como nível escolar. De modo geral, podese observar que o nível de escolaridade reflete o desenvolvimento das regiões onde estes estudos foram realizados.

O leite vendido informalmente e comercializado sem inspeção sanitária ou tratamento térmico adequado, no Brasil, é denominado "leite informal", comumente comercializados em utensílios plásticos ou de vidro (16), muito embora os riscos de consumir leite in natura sejam divulgados e de conhecimento público, estando estabelecidos os padrões de identidade e qualidade na legislação brasileira, na Instrução Normativa no 76, de 26 de novembro de 2018 (17).

Os resultados aqui descritos apontam que em Salvaterra e Soure a preferência pelo consumo de leite in natura é muito alta, visto que em Salvaterra, 57,5\% (96/167) consumiam 
leite fervido, 29,3\% (49/167) sem ferver, 10,8\% (18/167) UHT e 4,2\% (7/167) pasteurizado. Já em Soure, 55\% (126/229) relataram consumir leite fervido, 31\% (71/229) sem ferver, 9,6\% (22/229) UHT e 6,1\% (14/229) pasteurizado. Os resultados possuem uma séria implicação em Saúde Pública, já que o consumo desses produtos pode veicular uma série de doenças transmitidas por alimentos (DTA) (18), podendo acarretar sérios problemas de saúde coletiva e deve ser levada em consideração pelos órgãos fiscalizadores, para que possam coibir este tipo de comercialização. Contudo, vale ressaltar que essa é uma característica do perfil dos consumidores da região analisada.

O hábito de consumir leite in natura por uma parcela considerável da população está diretamente relacionado a conceitos previamente formados de que esse produto possui boa qualidade, além do desconhecimento dos riscos que esse produto pode oferecer (19), o que pode justificar o consumo elevado pelos entrevistados deste estudo. Esse fato revela que, possivelmente, a característica de consumir leite cru pode estar atrelada a realidade da população analisada. O arquipélago do Marajó, ao que parece, ainda enfrenta problemas de logística e deficiência de acesso à informação.

Em relação à origem da matéria-prima dos produtos lácteos, foi observado em Salvaterra que $21,2 \%$ (36/170) dos entrevistados preferiam leite e derivados de origem bovina, 26,5\% (45/170) de origem bubalina, e 52,3\% (89/170) consomem produtos originados de ambas as espécies. Já em Soure, 19,3\% (45/233) das pessoas entrevistadas preferiam os de origem bovina, 41,6\% (97/233) bubalina, e 39\% (91/233) consomem produtos de ambas as espécies. Esta predileção pode ser explicada pela disponibilidade dos produtos e por questões culturais da região, considerando que o primeiro território brasileiro onde esta espécie animal habitou foi o arquipélago do Marajó (18). Apesar de em Salvaterra o consumo de produtos de ambas as espécies tenha sido mais expressivo, em Soure houve preferência pelo consumo de produtos lácteos bubalinos, o que pode estar relacionado à disponibilidade comercial ou ao preço. Esse fato pode ser corroborado pelo trabalho de Zanela et al. (20), que verificaram nas feiras da região Sul do Brasil que 42,49\% dos entrevistados consumiam produtos de origem bubalina e que esses consumiriam mais produtos se houvesse maior disponibilidade no mercado, preço mais acessível e hábito de consumo.

Grande parte dos entrevistados em Salvaterra, quando questionados quanto à percepção das diferenças entre matéria-prima bovina e bubalina (Tabela 2), 68,2\% (116/170) afirmaram não saber distinguir entre o leite bubalino e o bovino, em Soure a maioria disse que sim, representando 64,4\% (150/233). Estes achados contrastam, em certos aspectos, com os relatos de Tonhati et al. (21), para os quais o leite de búfala possui características que possibilitam fácil identificação dos pontos de vista físico-químico e organoléptico, como o sabor ligeiramente adocicado e coloração mais branca do que o leite bovino. Quanto à detecção de diferença da matéria-prima em queijo, em Soure não houve diferença entre as respostas, porém em Salvaterra, 64,12\% (109/170) dos entrevistados disseram estar cientes da diferença entre as duas origens dos produtos.

Quanto à possibilidade do consumo de produtos mistos, os habitantes do município de Soure se mostraram mais relutantes do que os de Salvaterra no que se referiu ao consumo de leite. Já quando a compra se referia ao queijo, não se observou diferença entre os municípios, sendo a resposta "não" a mais frequente (Tabela 3).

Quando os entrevistados dos dois municípios foram questionados sobre a segurança alimentar, inspeção, fiscalização, embalagem, conservação e comercialização dos produtos lácteos, $75,2 \%$ (303/403) do total de pessoas consultadas afirmaram que o consumo de produtos lácteos pode causar alguma doença e 24,8\% (100/403) disseram que não. Já quando questionados sobre quais seriam as enfermidades veiculadas por esses produtos, a brucelose foi citada por $0,9 \%$ (4/403). Ainda, 27\% (109/403) dos entrevistados relataram que já haviam ficado doentes, ou tiveram alguém de sua família acometido de alguma enfermidade ao

Cruz AV, Silva EAC, Lima ALA, Jesus IS, Feitosa RF, Dufossé MCS, et al. Perfil sociodemográfico e percepção dos consumidores sobre a segurança alimentar de produtos lácteos de origens bubalina e bovina em municípios do Arquipélago do Marajó, estado do Pará. Vet. e Zootec. 2020.; 27: 001-014. 
consumir leite ou algum derivado, enquanto $66,5 \%$ responderam não, e $6,5 \%$ que não saberia afirmar (Tabela 4). Considerando o fato de que boa parte dos entrevistados disse consumir leite in natura, fica evidente a necessidade de informações concretas sobre as DTAs chegue até os consumidores. Desse modo, é provável que passem a observar as boas práticas seguidas por estabelecimentos comerciantes de alimentos e contribuam para a diminuição dos possíveis casos de contaminações e surtos (22).

Tabela 2. Distribuição das respostas dos habitantes dos municípios de Soure e Salvaterra sobre a capacidade de identificar a diferença entre produtos lácteos de origem bovina e bubalina.

\begin{tabular}{|c|c|c|c|c|c|}
\hline Pergunta & & & cípio & & \multirow[b]{3}{*}{ p valor } \\
\hline \multirow{2}{*}{$\begin{array}{c}\text { Consegue identificar a } \\
\text { diferença entre... }\end{array}$} & & Soure & Salvaterra & \multirow[b]{2}{*}{ Chi $^{2}$} & \\
\hline & & n $(\%)$ & n $(\%)$ & & \\
\hline \multirow{2}{*}{ Leite bovino do bubalino? } & Sim & $150(64,38)$ & $54(31,76)$ & \multirow{2}{*}{22.03} & \multirow{2}{*}{$>0,005$} \\
\hline & Não & $83(35,62)$ & $116(68,24)$ & & \\
\hline \multirow{2}{*}{$\begin{array}{c}\text { Queijo bovino e } \\
\text { bubalino? }\end{array}$} & Sim & $114(48,93)$ & $109(64,12)$ & \multirow{2}{*}{5.26} & \multirow{2}{*}{$>0,05$} \\
\hline & Não & $119(51,07)$ & $61(35,88)$ & & \\
\hline
\end{tabular}

Tabela 3. Distribuição das respostas dos habitantes dos municípios de Soure e Salvaterra sobre se compraria leite e queijo bubalino sabendo que contem leite bovino.

\begin{tabular}{|c|c|c|c|c|c|}
\hline \multirow{3}{*}{$\begin{array}{c}\text { Pergunta } \\
\text { Compraria se soubesse } \\
\text { que contem... }\end{array}$} & & \multicolumn{2}{|c|}{ Município } & \multirow[b]{3}{*}{$\mathrm{Chi}^{2}$} & \multirow[b]{3}{*}{ p valor } \\
\hline & & Soure & Salvaterra & & \\
\hline & & n (\%) & $\mathrm{n}(\%)$ & & \\
\hline \multirow{2}{*}{$\begin{array}{l}\text { Leite bubalino contém } \\
\text { leite bovino? }\end{array}$} & Sim & $81(34.76)$ & $93(54.71)$ & \multirow{2}{*}{8.18} & \multirow{2}{*}{$>0.005$} \\
\hline & Não & $152(65.24)$ & 77 (45.29) & & \\
\hline \multirow{2}{*}{$\begin{array}{c}\text { Queijo bubalino contém } \\
\text { leite bovino? }\end{array}$} & Sim & $92(39.48)$ & $64(37.65)$ & \multirow{2}{*}{0.13} & \multirow{2}{*}{0.77} \\
\hline & Não & $141(60.52)$ & $106(62.35)$ & & \\
\hline
\end{tabular}

Tabela 4. Percepção dos consumidores sobre a segurança alimentar em relação as enfermidades transmitidas por produtos lácteos nos municípios de Salvaterra e Soure, estado do Pará.

\begin{tabular}{|c|c|c|c|c|c|}
\hline \multirow{2}{*}{ Pergunta } & \multirow{2}{*}{ Alternativa } & \multicolumn{2}{|c|}{ Município } & \multirow{2}{*}{ Chi } & \multirow{2}{*}{$p$ valor } \\
\hline & & Soure & Salvaterra & & \\
\hline \multirow{2}{*}{$\begin{array}{l}\text { Ouviu falar em contaminação de } \\
\text { alimentos? }\end{array}$} & Sim & $205(87,98)$ & $145(85,29)$ & \multirow{2}{*}{0,1771} & \multirow{2}{*}{0,67388} \\
\hline & Não & $28(12,02)$ & $25(14,71)$ & & \\
\hline \multirow{2}{*}{$\begin{array}{l}\text { Consumo de leite e derivados } \\
\text { pode causar alguma doença? }\end{array}$} & Sim & $172(85,29)$ & $131(87,98)$ & \multirow{2}{*}{0,44} & \multirow{2}{*}{0,50712} \\
\hline & Não & $61(14,71)$ & $39(12,02)$ & & \\
\hline \multirow{3}{*}{$\begin{array}{l}\text { Você ou alguém da sua casa já } \\
\text { ficou doente após beber leite ou } \\
\text { consumir algum derivado do } \\
\text { leite? }\end{array}$} & Sim & $59(25,32)$ & $50(29,41)$ & \multirow{3}{*}{0,99863} & \multirow{3}{*}{0,60695} \\
\hline & Não & $161(69,1)$ & $107(69,94)$ & & \\
\hline & Não sei & $13(5,58)$ & $13(7,65)$ & & \\
\hline
\end{tabular}

Tendo em vista o armazenamento de alimentos, os entrevistados foram perguntados quanto à verificação das embalagens dos produtos lácteos no momento da compra, 72,2\% (291/403) de ambos os municípios disseram observar e 27,8\% (112/403) que não observavam. Sobre a percepção dos locais de compra, 18,6\% (75/403) consideraram os locais excelentes, 73,7\% (297/403) em boas condições e 7,7\% (31/403) em condição ruim (Tabela 5). Apesar dos resultados apresentados, alguns autores como Seixas et al. (23) relatam a contaminação 
em produtos lácteos comercializados no Arquipélago, ao realizarem análises físico-químicas e microbiológicas de queijos do Marajó tipo manteiga, observaram contagens de Staphylococcus aureus fora dos padrões da norma vigente. O que pode estar relacionado às más condições de armazenamento dos produtos, considerando que este tipo de queijo passa por cozimento da massa durante o processo de obtenção.

Tabela 5. Percepção dos consumidores sobre a segurança alimentar em relação à inspeção e fiscalização dos produtos lácteos nos municípios de Salvaterra e Soure, estado do Pará.

\begin{tabular}{|c|c|c|c|c|}
\hline \multirow{2}{*}{ Pergunta } & \multirow{2}{*}{ Alternativa } & \multicolumn{2}{|c|}{ Município n (\%) } & \multirow{2}{*}{ Chi } \\
\hline & & Soure & Salvaterra & \\
\hline \multirow{2}{*}{$\begin{array}{l}\text { Quando você compra produto, observa a } \\
\text { embalagem? }\end{array}$} & Sim & $\begin{array}{c}177 \\
(75,97)\end{array}$ & $114(67,06)$ & \multirow{2}{*}{1,5936} \\
\hline & Não & $56(32,94)$ & $56(24,03)$ & \\
\hline \multirow{2}{*}{$\begin{array}{c}\text { Conhece os órgãos de fiscalização de } \\
\text { alimentos? }\end{array}$} & Sim & $98(42,06)$ & $55(32,35)$ & \multirow{2}{*}{2,1578} \\
\hline & Não & $135(57,94)$ & $115(67,65)$ & \\
\hline \multirow{2}{*}{ Conhecem as siglas SIF, SIE e SIM? } & Sim & $59(25,32)$ & $30(17,65)$ & \multirow{2}{*}{1,9341} \\
\hline & Não & $174(74,68)$ & $140(82,35)$ & \\
\hline \multirow{2}{*}{$\begin{array}{l}\text { Procura uma dessas siglas nas embalagens de } \\
\text { leite ou derivados? }\end{array}$} & Sim & $38(16,32)$ & $23(13,53)$ & \multirow{2}{*}{0,3636} \\
\hline & Não & $195(83,69)$ & $147(86,47)$ & \\
\hline \multirow{3}{*}{$\begin{array}{c}\text { Como percebe as condições de higiene do local } \\
\text { de vendas de leite e derivados? }\end{array}$} & Excelente & $51(21,89)$ & $24(14,12)$ & \multirow{3}{*}{3,1229} \\
\hline & Boa & $169(72,53)$ & $128(75,29)$ & \\
\hline & Ruim & $13(5,58)$ & $18(10,59)$ & \\
\hline \multirow{2}{*}{ Você já ouviu falar em fraude alimentar? } & Sim & $133(78,11)$ & $182(78,24)$ & \multirow{2}{*}{0} \\
\hline & Não & $37(21,89)$ & $51(21,76)$ & \\
\hline \multirow{2}{*}{ Você já foi vítima de fraude alimentar? } & Sim & $56(24,03)$ & $48(28,24)$ & \multirow{2}{*}{0,41728} \\
\hline & Não & $177(75,97)$ & $122(71,76)$ & \\
\hline
\end{tabular}

Os produtos de origem animal industrializados no Brasil recebem um selo de inspeção, certificando que o alimento não oferece risco para a população (24). O serviço de Inspeção de POA no Brasil está distribuído em três esferas, federal, estadual e municipal. Uma vez questionados se conheciam os principais órgãos de fiscalização brasileiros e as siglas SIF (Serviço de Inspeção Federal), SIE (Serviço de Inspeção Estadual) e SIM (Serviço de Inspeção Municipal), 62\% (250/403) relataram não conhecer, enquanto 38\% (153/403) afirmaram ter conhecimento. Estes dados demonstram que há uma falta de divulgação ou atuação efetiva desses órgãos, talvez pela falta de mão de obra, devido à dificuldade de acesso a essas localidades, o que também pode estar relacionado ao comércio informal de produtos na região.

Além disso, as respostas apontam a falta de instrução dos entrevistados quanto à diferença entre os alimentos fiscalizados e informais. Em relação às siglas SIF, SIE e SIM, o resultado observado foi de $78 \%$ (314/403) não conheciam e 22,1\% (89/403) para os que conheciam e $85 \%$ (342/403) disseram que não levar em consideração as siglas no momento da compra, enquanto $15,1 \%$ (61/403) afirmaram considerar. Este fato também foi observado por Vidal-Martins et al. (13) que verificaram que embora 40,12\% reconhecessem a sigla SIF, poucos souberam dizer sobre as demais. Já Fanalli (25), ao analisar as opiniões de determinada população com relação à segurança alimentar e às implicações a saúde pública, observou que $12,28 \%$ não sabiam o significado da sigla SIF.

No que se refere ao conhecimento sobre fraude (Tabela 5), 78,2\% (315/403) dos indivíduos já haviam ouvido falar em fraude alimentar e $22 \%$ (88/403) relataram não ter 
conhecimento sobre o tema. Os entrevistados foram inquiridos também se já haviam sido vítimas de fraude, 26\% (104/403) declararam que sim e 74,2\% (299/403) que não. Os casos de fraude citados foram adições de água ao leite. Embora ações fraudulentas possam ocorrer sem que o consumidor perceba, esses resultados demonstram que é preciso promover a educação sanitária e ações concretas dos órgãos competentes que favoreçam o consumo de produtos inspecionados.

Com a análise de correspondência múltipla pode-se observar os possíveis agrupamentos entre as respostas obtidas no trabalho. As Figuras de 1 a 2 apresentam os resultados obtidos na ACM relacionadas ao perfil sociodemográfico dos consumidores apresentados na Tabela 1.

Em Soure, os dados obtidos pela ACM demonstraram que as características do perfil sociodemográfico dos entrevistados eram, em sua maioria, do sexo masculino, acima de 40 anos, preferiam consumir produtos lácteos de origem bubalina, comprados diretamente do produtor ou de ambulantes e feiras. Esses entrevistados apresentavam nível escolar entre ensino fundamental completo e incompleto (Figura 1). A preferência pelo consumo do leite de búfala por este grupo revela um conhecimento empírico dos entrevistados, pois a principal justificativa era de que o leite seria mais forte e de melhor qualidade nutricional.

A análise demonstrou, ainda, que o consumo de produtos lácteos de ambas as origens estavam atrelados às mulheres de 31 a 40 anos, com ensino médio completo, superior completo e incompleto, preferindo comprar em supermercados e em mais de uma opção de escolha do questionário (Figura 1). Dezani et al. (26) afirmam que as mulheres são mais frequentadoras de supermercados do que homens, o que pode estar atrelado, segundo Souza et al. (27), ao fato de mulheres buscarem se alimentar de forma mais equilibrada. Além disso, muitas vezes na divisão do trabalho as mulheres assumem a responsabilidade das atividades domésticas, mesmo sendo a população em sua maioria ser compostas por homens (5).

Quanto ao consumo de produtos lácteos de origem bovina, observou-se relação entre pessoas de ambos os sexos, entre as faixas etárias menores de 20 anos e de 21 a 30 anos, as quais adquirem os produtos no mercado municipal, supermercados e padaria (Figura 1). Quanto ao consumo de produtos lácteos de origem bovina observou-se relação entre pessoas de ambos os sexos, entre as faixas etárias menores de 20 anos e 21 a 30 anos, as quais adquirem os produtos no mercado municipal, supermercados e padarias. Esse dado pode estar relacionado ao fato de que a maior parte dos produtos industrializados serem de origem bovina, estando assim mais disponíveis em mercados formais.

A distribuição das variáveis em Salvaterra foi similar à encontrada em Soure, uma vez que o consumo de produtos lácteos bubalinos é realizado por pessoas acima de 40 anos, com nível escolar fundamental completo e incompleto, sendo adquiridos direto do produtor ou por intermédio de vendedores locais, onde os comerciantes são responsáveis pela produção de produtos como queijo, coalhada, doce de leite, iogurte e manteiga. A preferência por produtos bovinos foi evidenciada principalmente por mulheres, menores de 20 anos, com ensino médio completo e incompleto que escolheram como opção mais de um local de compra. Já o consumo de produtos lácteos de ambas as espécies animais, foi caracterizado também por pessoas do sexo feminino, com nível escolar superior completo e incompleto e idade entre 21 a 30 anos, os quais adquirem estes produtos em mais de uma opção de local de compra (Figura 2). 


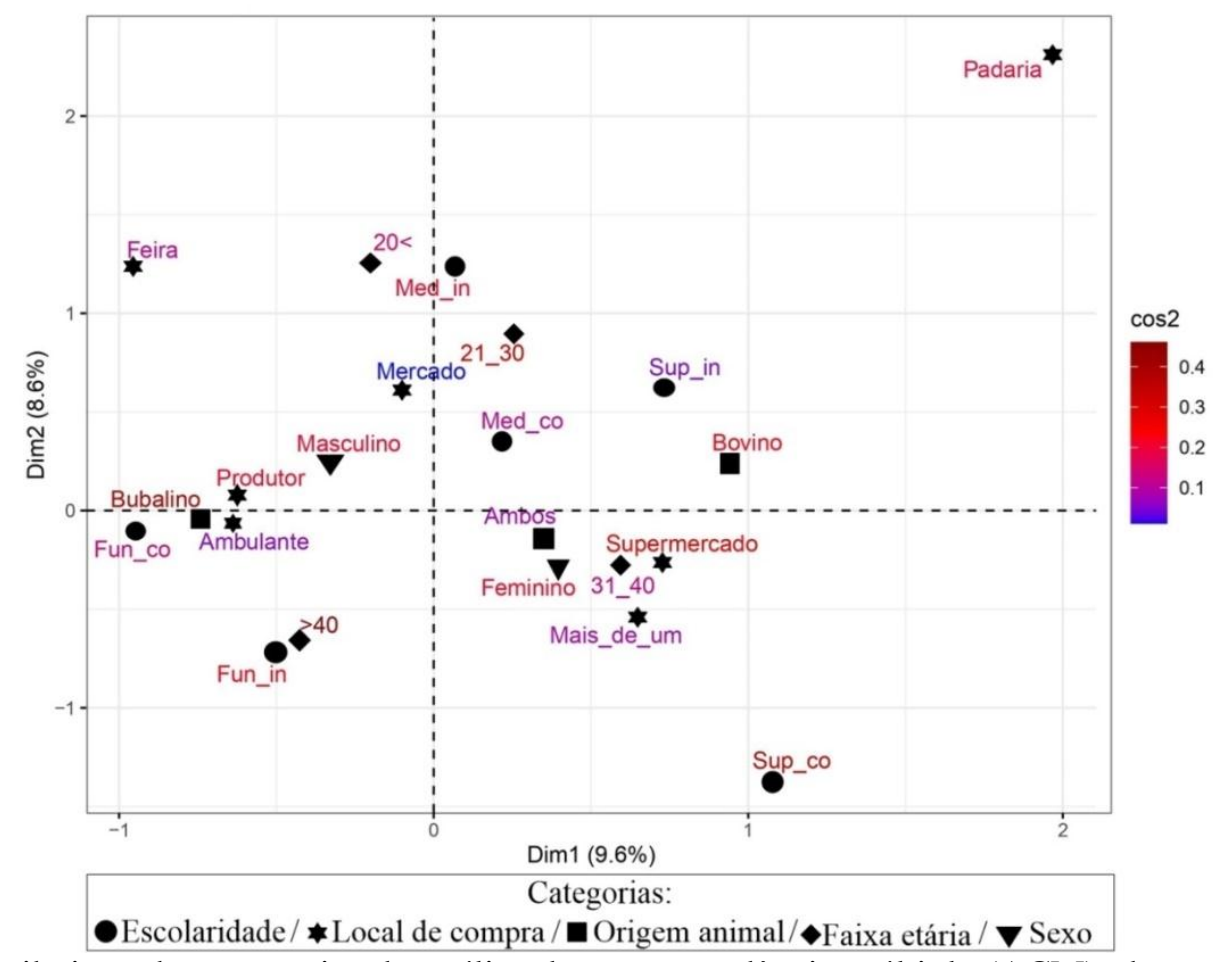

Figura 1. Distribuição das categorias da análise de correspondência múltipla (ACM), de acordo com as respostas obtidas em Soure quanto aos aspectos sociodemográficos dos entrevistados. Fun_co: Fundamental completo; Fun_in: Fundamental incompleto; Med_in: Ensino médio incompleto; Med_co: Ensino médio completo; Sup_in: Superior incompleto; Sup_co: Superior completo.

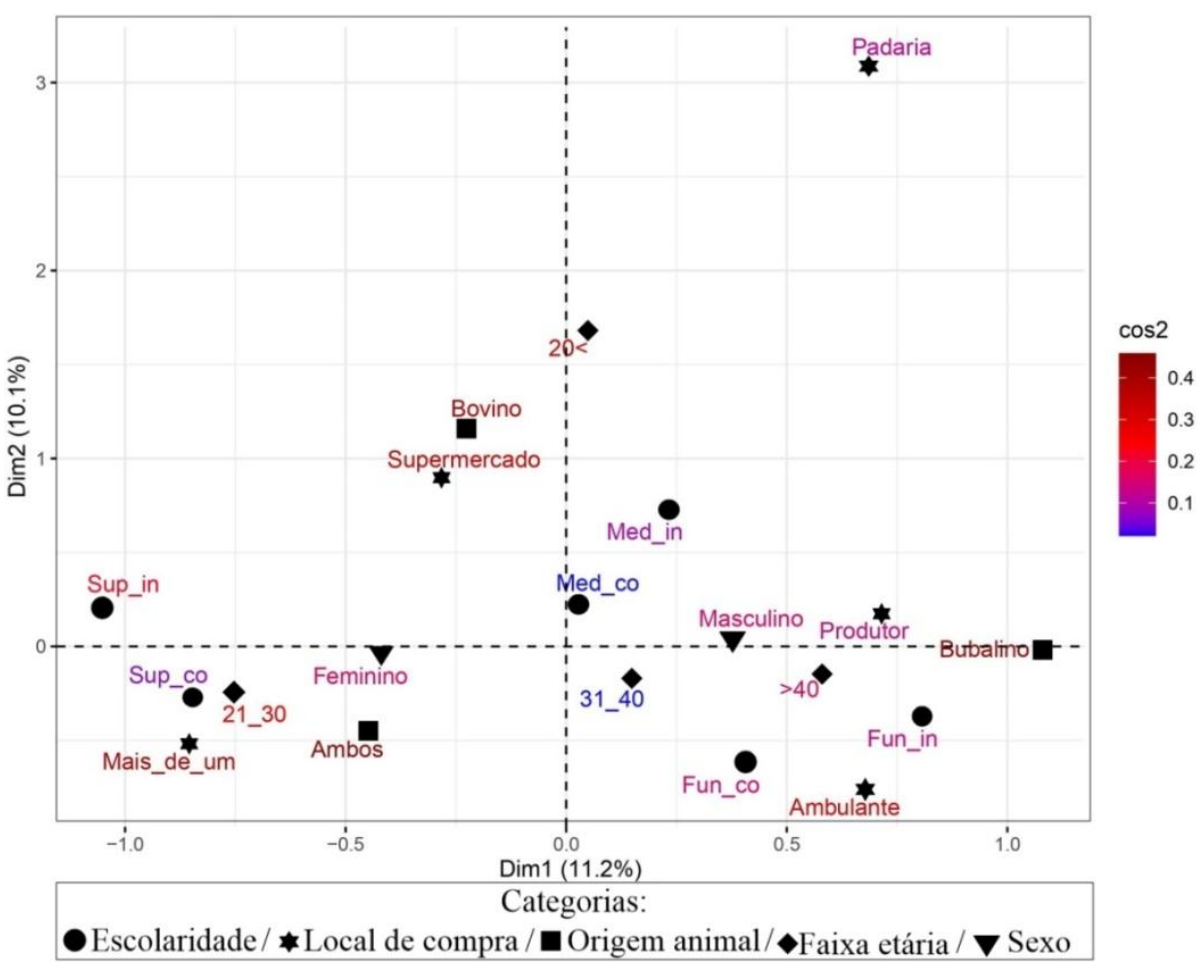

Figura 2. Distribuição das categorias de análise de correspondência múltipla (ACM) de acordo com as respostas obtidas em Salvaterra quanto aos aspectos sociodemográficos dos entrevistados. Fun_co: Fundamental completo; Fun_in: Fundamental incompleto; Med_in: Ensino médio incompleto; Med_co: Ensino médio completo; Sup_in: Superior incompleto; Sup_co: Superior completo.

Cruz AV, Silva EAC, Lima ALA, Jesus IS, Feitosa RF, Dufossé MCS, et al. Perfil sociodemográfico e percepção dos consumidores sobre a segurança alimentar de produtos lácteos de origens bubalina e bovina em municípios do Arquipélago do Marajó, estado do Pará. Vet. e Zootec. 2020;; 27: 001-014. 
A ACM também foi realizada visando relacionar o perfil dos consumidores e sua capacidade para distinguir matérias-primas bovina e bubalina (Figura 3 A e B).
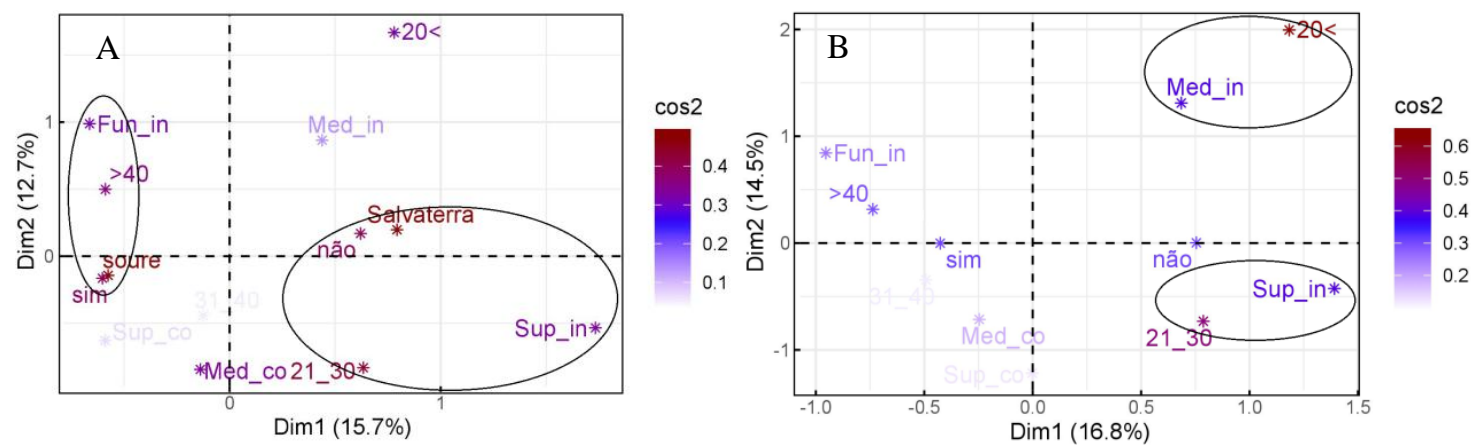

Figura 3. Análise correspondência múltipla (ACM). (A) perfil de entrevistados que se denominam capazes ou não de distinguir entre leite bovino e bubalino nos municípios de Soure e Salvaterra; (B) perfil de entrevistados que se denominam capazes ou não de distinguir a diferença entre queijo bovino e bubalino nos municípios de Salvaterra.

Nesse caso, os entrevistados em Soure que disseram saber a diferença entre o leite bovino e bubalino tinham idade acima de 40 anos e ensino fundamental incompleto, enquanto os entrevistados de Salvaterra que disseram não saber a diferença entre os produtos estavam na maioria dos casos na faixa etária de 21 a 30 anos, com superior incompleto (Figura 3A). Quando perguntado se sabiam a diferença entre o queijo de leites bovino e bubalino, não houve diferença entre sim e não no município de Soure (Tabela 2), porém em Salvaterra as pessoas que disseram saber a diferença foram agrupadas em dois grupos, um com faixa etária entre 21 a 30 anos, com ensino superior incompleto, e outro com pessoas menores de 20 anos e ensino médio incompleto (Figura 3B).

Por fim, o perfil dos consumidores que comprariam ou não leite e queijos mistos, contendo matéria-prima bovina e bubalina (Figuras 4A e B). Os resultados de Soure para quem respondeu não para a questão "Você compraria leite bubalino que contém leite bovino?" eram de pessoas com ensino fundamental incompleto e acima de 40 anos. Já as que disseram sim deram origem a dois grupos: um com faixa etária entre 21 a 30 anos, que possuía ensino médio completo e superior incompleto, e outro com pessoas menores de 20 anos, com ensino médio incompleto (Figura 4A). Estes resultados demonstram uma possível relação entre a faixa etária e a decisão de consumir ou não o leite misto.
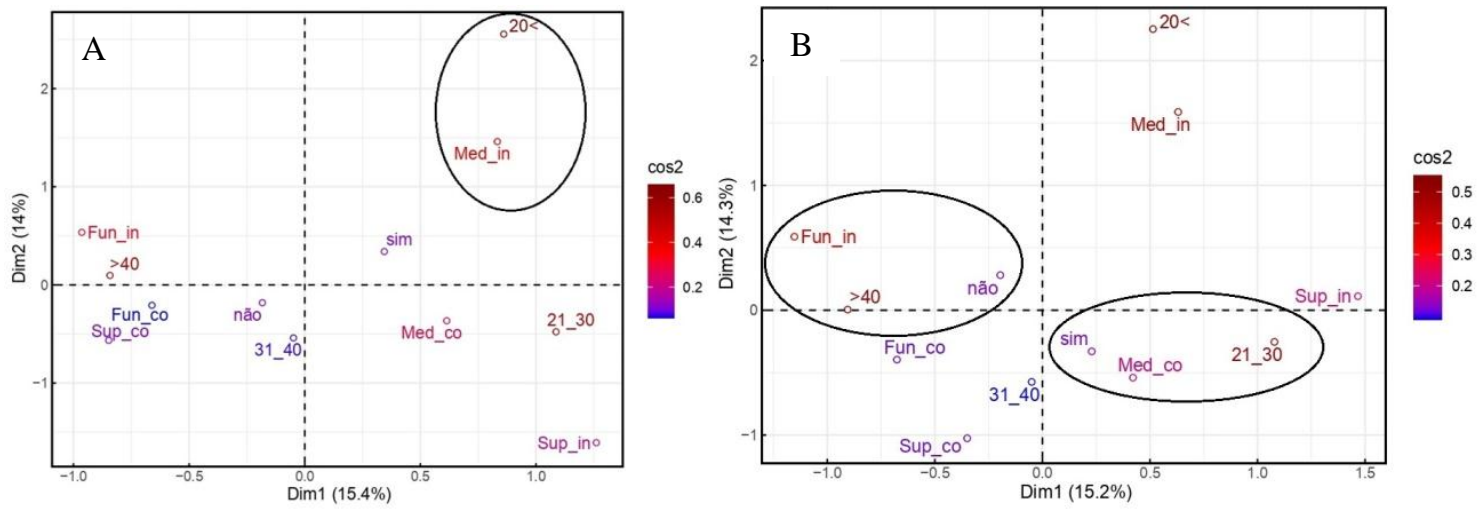

Figura 4. Análise de correspondência múltipla (ACM) do perfil de entrevistados quanto a possibilidade de consumir ou não leite bubalino que contém leite bovino nos municípios de Soure (A) - Possibilidade de consumir queijo misto nos municípios de Soure e Salvaterra (B).

Cruz AV, Silva EAC, Lima ALA, Jesus IS, Feitosa RF, Dufossé MCS, et al. Perfil sociodemográfico e percepção dos consumidores sobre a segurança alimentar de produtos lácteos de origens bubalina e bovina em municípios do Arquipélago do Marajó, estado do Pará. Vet. e Zootec. 2020.; 27: 001-014. 
Para quem disse sim para questão "Você compraria queijo sabendo que contém leite bubalino e leite bovino", considerou-se os dois municípios pelo fato de não haver diferença entre as respostas (Tabela 3), também houve a formação de grupos, onde é observado que o nível escolar ficou entre ensino médio completo e superior incompleto, com faixa etária de 21 a 30 anos. Para quem não consumiria queijo misto, os entrevistados apresentaram escolaridade do fundamental incompleto ao superior completo, com idades de 31 anos a 40 anos ou mais (Figura 4B). Nesse contexto, a maior parte da variabilidade no comportamento do consumidor está relacionada a características individuais (28).

\section{CONCLUSÃO}

Observou-se que os entrevistados que fizeram parte deste estudo mantiveram um padrão, apresentando o mesmo ponto de vista sobre as questões abordadas, também houve similaridade em relação aos perfis demográficos das duas cidades. De modo geral, há uma predileção por produtos de origem bubalinas, o que confere uma característica cultural única ao povo Marajoara, no entanto foi possível identificar dois principais grupos, onde os consumidores de produtos essencialmente bubalinos são pessoas mais velhas, com nível escolar básico, que optam por comprar diretamente do produtor ou de cooperativas. Por outro lado, o consumo de produtos lácteos, tanto bubalinos quanto bovinos, foi notado entre pessoas mais jovens, adquiridos, sobretudo em estabelecimentos comerciais.

\section{REFERÊNCIAS}

1. Guerrero L, Colomer Y, Guàrdia Md, Xicola J, Clotet R. Consumer attitude towards store brands. Food Qual Prefer. 2000;11(5):387-95. doi: https://doi.org/10.1016/S09503293(00)00012-4.

2. Oliveira FFO. Comportamento do consumidor: um estudo das teorias de marketing aplicadas ao turismo. Saberes Interdiscip. 2008;1(2):53-78.

3. Olsen So, Heide M, Dopico Dc, Toften K. Explaining intention to consume a new fish product: a cross-generational and cross-cultural comparison. Food Qual Prefer. 2008;19(7):618-27. doi: https://doi.org/10.1016/j.foodqual.2008.04.007.

4. Kendall H, Kuznesofa S, Dean M, Chan My, Clark B, Home R, et al. Chinese consumer's attitudes, perceptions and behavioural responses towards food fraud. Food Control. 2019;95(1):339-51. doi: https://doi.org/10.1016/j.foodcont.2018.08.006.

5. Matiuzzo AG, Silva AM. Caracterização do consumo de leite no município de Campos de Júlio - MT. Nucleus Anim. 2018;10(2):97-105. doi: http://dx.doi.org/10.3738/21751463.3019.

6. Ferreira AGG, Lyra DG, Silva JCS, Soares FMF, Araújo CA. Perfil dos consumidores de leite bovino in natura no município de Santana do Ipanema - Alagoas. Nutritime Rev Eletronica [Internet]. 2017 [cited 2020 Apr 10];14(4):6056-65. Available from: https://docplayer.com.br/52386045-Perfil-dos-consumidores-de-leite-bovino-in-natura-nomunicipio-de-santana-do-ipanema-alagoas.html 
7. Marques Css, Oaigen RP, Moraes CM, Santos MAS, Lourenço Júnior, JB, Abel I. Perfil dos consumidores da carne de búfalo em Belém, Pará, Brasil. Acta Vet Bras. 2015;9(2):126-133. doi: https://doi.org/10.21708/avb.2015.9.2.4662.

8. Instituto Brasileiro de Geografia e Estatística - IBGE [Internet]. Rio de Janeiro: IBGE; 2018 [cited 2019 Jul 2]. Available from: www.ibge.gov.br/cidades-e-estados/pa/soure.html

9. Agência de Defesa Agropecuária do Estado do Pará - ADEPARÁ. Estado do Pará é destaque na produção de queijo artesanal [Internet]. Belém: ADEPARÁ; 2017 [cited 2019 Apr 2]. Available from: http://adepara.pa.gov.br/artigos/estado-do-par\%C3\%A1$\% \mathrm{C} 3 \% \mathrm{~A} 9-$ destaque-na-produ\%C3\%A7\%C3\%A3o-de-queijo-artesanal

10. Oliveira CM, Mattos CAC, Santana AC. Aspectos produtivos e socioeconômicos do arranjo produtivo local bovino e bubalino no arquipélago do Marajó, estado do Pará. Rev Agron Ambient. 2016;9(1):25-45. doi: https://doi.org/10.17765/21769168.2016v9n1p25-45.

11. Brasil. Conselho Nacional de Saúde. Resolução CNS no 466, de 12 de Dezembro de 2012. Diário Oficial da União [Internet]. 13 jun 2013[cited 2019 Apr 2]; n. 12, sec. 2, p. 59. Available from: http://conselho.saude.gov.br/resolucoes/2012/Reso466.pdf

12. Dean AG, Sullivan KM, Soe MM. OpenEpi: Open Source Epidemiologic Statistics for Public Health, Versão [Internet]. Atlanta; 2013 [citado 2018 Oct 10]. Available from: www.OpenEpi.com

13. Vidal-Martins AMC, Bürger KP, Gonçalves ACS, Grisólio APR, Aguilar CEG, Rossi GAM. Avaliação do consumo de leite e produtos lácteos informais e do conhecimento da população sobre os seus agravos à saúde pública, em um município do estado de São Paulo, Brasil. Bol Ind Anim. 2013;70(3):221-7. doi: 10.17523/bia.v70n3p221.

14. Pereira GHS, Bankuti FI, Brito MM, Pozza MSS, Damasceno JC. Perfil do consumidor de leite e derivados no munícipio de Maringá, PR. Sci Agrar Parana. 2018;17(1):44-54. doi: http://dx.doi.org/10.18188/sap.v17i1.16254.

15. Marciana MV, Marciano MV, Rauecker UM, Coelho KO. Consumo e acesso de produtos lácteos sem lactose. Rev UNINGÁ [Internet]. 2019 [cited 2019 Apr 2];56(1):58-65. Available from: http://revista.uninga.br/index.php/uninga/article/view/2139

16. Motta RG. Indicadores de qualidade e composição de leite informal comercializado na região Sudeste do Estado de São Paulo. Pesqui Vet Bras. 2015;35(5):417-23.

17. Brasil. Ministério da Agricultura, Pecuária e Abastecimento. Instrução Normativa $n^{\circ} 76$, de 26 de Novembro de 2018. Diário oficial da união [Internet]. 30 Nov 2018 [cited 2020 Feb 11]; sec. 1, p.9. Available from: http://www.in.gov.br/materia//asset_publisher/Kujrw0TZC2Mb/content/id/52750137/do1-2018-11-30-instrucaonormativa-n-76-de-26-de-novembro-de-2018-52749894IN\%2076

18. Lourenço Júnior JB, Garcia AR. Panorama da bubalinocultura na Amazônia [Internet]. In: 1o Encontro Internacional da Pecuária da Amazônia; 2008; Belém, PA. Belém: FAEPA, Instituto Frutal, SEBRAE-PA; 2008 [cited 2020 Feb 11]. Available from: http://www.alice.cnptia.embrapa.br/alice/handle/doc/409969 
19. Silveira MLR, Bertagnolli SMM. Avaliação da qualidade do leite cru comercializado informalmente em feiras livres no município de Santa Maria/ RS. Vigil Sanit Debate [Internet]. 2014 [cited 2019 Apr 2];2(2):75-80. Available from: https://visaemdebate.incqs.fiocruz.br/index.php/visaemdebate/article/view/135

20. Zanela MB, Kolling GJ, Fernandez VNV. Caracterização do perfil do consumidor de leite de búfala em feiras no Rio Grande do Sul [Internet]. Pelotas, RS: Embrapa; 2015 [cited 2019 Apr 2]. (Circular técnica n. 163). Available from: http://www.infoteca.cnptia.embrapa.br/infoteca/handle/doc/1047446

21. Tonhati H, Seno Lo, Mendoza-Sanchez G, Aspilcueta-Borquis RR. Qualidade do leite de búfalas e correlações entre a produção e seus principais constituintes. Rev Inst Laticin Cândido Tostes [Internet]. 2005 [cited 2019 Apr 2]. Available from: https://www.researchgate.net/publication/273764688_qualidade_do_leite_de_bufalas_e_ correlacoes_entre_a_producao_e_seus_principais_constituintes

22. Leal D. Crescimento da alimentação fora do domicílio. Segur Aliment Nutr. 2010;17(1):123-32. doi: https://doi.org/10.20396/san.v17i1.8634806.

23. Seixas VNC, Félix MR, Silva GM, Perrone IT, Carvalho AF. Caracterização do queijo do Marajó tipo manteiga produzido em duas estações do ano. Cienc Rural. 2015;45(4):7306. doi: https://doi.org/10.1590/0103-8478cr20140463.

24. Pissolato B, Elesbão I. Adesão do serviço de inspeção municipal (SIM) ao sistema brasileiro de inspeção de produtos de origem animal (SISBI-POA): análise dos efeitos provocados no município de marau/rs. Rev GEDECON. 2018;6(2):1-14.

25. Fanalli S1. Perfil de consumo e percepção dos consumidores de carne: consequências sobre a saúde pública. Rev Cient Med Vet [Internet]. 2018 [cited 2019 Apr 2];15(31):113. Available from: http://faef.revista.inf.br/imagens_arquivos/arquivos_destaque/7YgU5DLnagIDsVr_20189-19-8-41-24.pdf

26. Dezani AA, La Retondo BB, Waideman MA. Determinantes de consumo dos produtos derivados do leite. Rev Eletronica Adm [Internet]. 2015 [cited 2019 Apr 2];14(1):62-84. Available from: http://periodicos.unifacef.com.br/index.php/rea/article/view/824/811

27. Souza APB, Fuke G, Nornberg JL. Fatores que influenciam a compra e conhecimento sobre propriedades funcionais de produtos lácteos. Discipl Sci Ser Cienc Saude [Internet]. 2013 [cited 2019 Apr 2];14(2):273-84. Available from: https://periodicos.ufn.edu.br/index.php/disciplinarumS/article/view/1055/999

28. Mendes CG, Sakatomo SM, Silva JBA, Jácome CGM, Leite AI. Análise físico-químicas e pesquisa de fraude no leite informal comercializado no município de Mossoró, RN. Cienc Anim Bras. 2010;11(2):349-56. doi: 10.526/cab.v11i2.1146.

Recebido em: 19/05/2020 Aceito em: 19/11/2020 